Monolithic high-temperature superconducting heterodyne Josephson frequency downconverter

J. Du, T. Zhang, J. C. Macfarlane, Y. J. Guo, and X. W. Sun

Citation: Appl. Phys. Lett. 100, 262604 (2012); doi: 10.1063/1.4731878

View online: http://dx.doi.org/10.1063/1.4731878

View Table of Contents: http://aip.scitation.org/toc/apl/100/26

Published by the American Institute of Physics

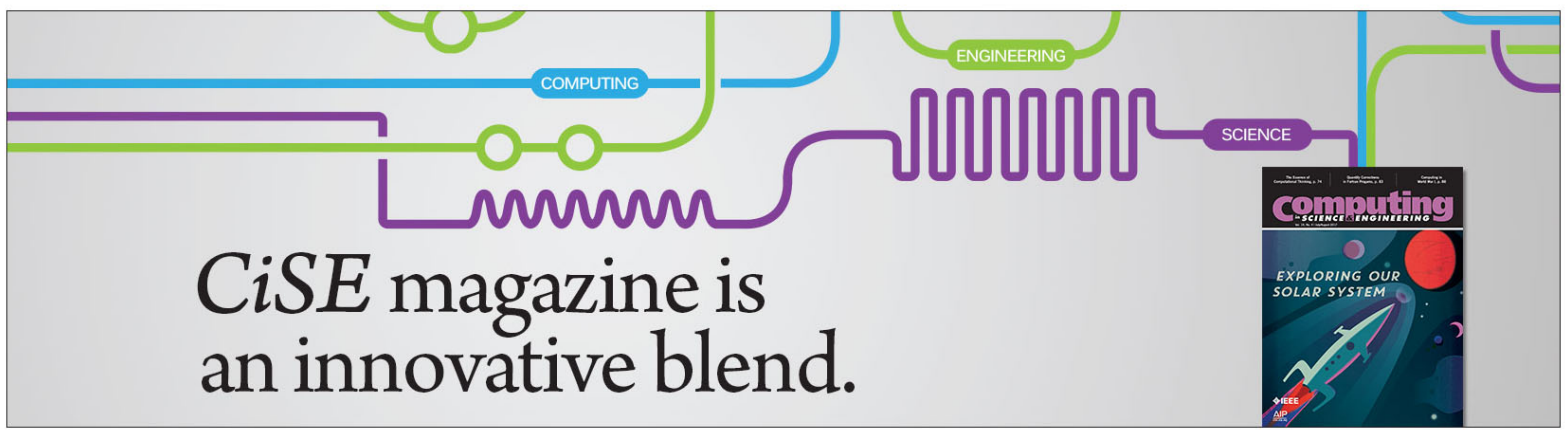




\title{
Monolithic high-temperature superconducting heterodyne Josephson frequency down-converter
}

\author{
J. Du, ${ }^{1}$ T. Zhang, ${ }^{2,3}$ J. C. Macfarlane, ${ }^{1}$ Y. J. Guo, ${ }^{2}$ and X. W. Sun ${ }^{3}$ \\ ${ }^{1}$ CSIRO Materials Science and Engineering, PO Box 218, Lindfield, NSW 2070, Australia \\ ${ }^{2}$ CSIRO ICT Centre, PO Box 76, Epping, NSW 1710, Australia \\ ${ }^{3}$ Shanghai Institute of Microsystem and Information Technology, CAS, China
}

(Received 14 May 2012; accepted 13 June 2012; published online 29 June 2012)

\begin{abstract}
A monolithic microwave integrated circuit (MMIC) frequency down-converter based on a compact high- $\mathrm{T}_{\mathrm{c}}$ superconducting (HTS) device is demonstrated. The on-chip integrated HTS down-converter consists of a 7-9 GHz bandpass filter for RF input, a lowpass filter for intermediate frequency output, and a self-pumped Josephson heterodyne mixer. All the above passive and active components are fabricated on a single $10 \mathrm{~mm} \times 20 \mathrm{~mm}$ chip of $\mathrm{YBa}_{2} \mathrm{Cu}_{3} \mathrm{O}_{7-\mathrm{x}}$ film on $\mathrm{MgO}$ substrate. Characterization of this MMIC HTS down-converter in terms of frequency response, conversion gain, frequency-tuneability, bias dependence, dynamic range, linearity, and intrinsic noise are presented in this paper. [http://dx.doi.org/10.1063/1.4731878]
\end{abstract}

High- $\mathrm{T}_{\mathrm{c}}$ superconducting (HTS) thin films have surface resistance values 10 to 100 times lower than that of conventional metallic films (copper or gold) at microwave frequencies. Microwave components and circuits made of HTS materials have many distinct advantages over their metallic counterparts, such as low insertion loss, high Q factor, low noise, low power consumption, and the potential for compact structure (due to high dielectric constant substrates). Highperformance HTS passive devices like filters and resonators have been well developed and applied in wireless network base stations. ${ }^{1-4}$ However, more sophisticated Josephson junction based microwave active devices, such as oscillators, amplifiers, and mixers (or detectors) have been slower to come to fruition, due to the rather challenging HTS Josephson junction technology compared to their low-temperature superconductor (LTS) counterparts. A hybrid configuration of HTS passive components with semi-conducting active devices has been typically employed in HTS RF front-end receiver circuits. ${ }^{1,2}$ Such a compromise makes systems bulky, inefficient, and unable to exploit the full potential of the superconductive components. An all-superconducting HTS integrated receiver system is a much desired technology.

Recently, we developed a gigahertz HTS Josephson heterodyne resistive superconducting quantum interference device (RSQUID) oscillator $^{5-7}$ and mixer $^{8-10}$ which demonstrated unique features of reduced oscillation linewidth (compared to a single Josephson junction device), wide-range frequency tuneability, and internally pumped frequency down-converting capability (no external LO needed). In the present work, we explore the possibility of combining a HTS RF band-pass filter (BPF) and a HTS low-pass filter (LPF) with the Josephson oscillator-mixer device to realise a monolithic HTS RF front-end receiver module. The aim is to improve the coupling efficiency between the HTS components, to achieve low loss, and to reduce both circuit size and power consumption. The work also demonstrates the possibility of achieving potentially more complex or larger scale high-performance HTS integrated circuits for terrestrial and space telecommunication systems.
Fig. 1 shows (a) a photograph of the packaged HTS monolithic microwave integrated circuit (MMIC) downconverter and (b) a close-up view of the junction area of the RSQUID heterodyne oscillator-mixer where two Josephson junctions are shunted by a thin-film Au resistor. The inset in Fig. 1(b) shows a scanning electron microscope photograph of the $\sim 2 \mu \mathrm{m}$ wide step-edge $\mathrm{YBa}_{2} \mathrm{Cu}_{3} \mathrm{O}_{7-\mathrm{x}}$ (YBCO) junction on a $\mathrm{MgO}$ substrate. A five-order half-wavelength resonator hairpin lines BPF is designed for the RF input. The resonance frequency of each resonator is around $8 \mathrm{GHz}$, and the passband of the filter is chosen to be from $7 \mathrm{GHz}$ to $9 \mathrm{GHz}$, which is used for many telecommunication services in Australia. A very compact step-impedance LPF is designed for the intermediate frequency (IF) output port allowing transmission of only the down-converted IF $(0-4 \mathrm{GHz})$. The filters are designed and modelled using Ansoft high frequency structure simulator (HFSS). The simulation and measurement of the filters will be reported elsewhere. The active device consists of two Josephson junctions connected via a small resistor $R_{\mathrm{S}}$ in an otherwise superconducting loop (see Fig. 1(b)). Under suitable conditions, it can be operated as a current-controlled heterodyne oscillator ${ }^{5-7}$ and a self-pumped mixer. ${ }^{8-10}$ Since the LO is generated intrinsically within the mixer structure itself, much more efficient coupling of the pumping power to the mixer is achieved despite the fact that the output power of a single Josephson oscillator is very small. Moreover, the frequency tuneability of the internal LO allows for in-situ adjustment of the down-converted intermediate frequency (IF) signal, which is a very useful feature in broadband front-end receivers.

Fig. 2 shows the schematic diagram of the MMIC frequency down-converter module and measurement set-up. The larger dashed box includes all the on-chip components and the smaller dashed box shows the schematic circuit of the HTS heterodyne RSQUID oscillator-mixer. Two dc currents, $I_{\mathrm{B}}$ and $I_{\mathrm{R}}$, are applied to bias the junctions and to control the current passing through the resistor $R \mathrm{~s}$, respectively. At the optimal biasing $\left(I_{\mathrm{B}}\right)$ point, the individual junctions 

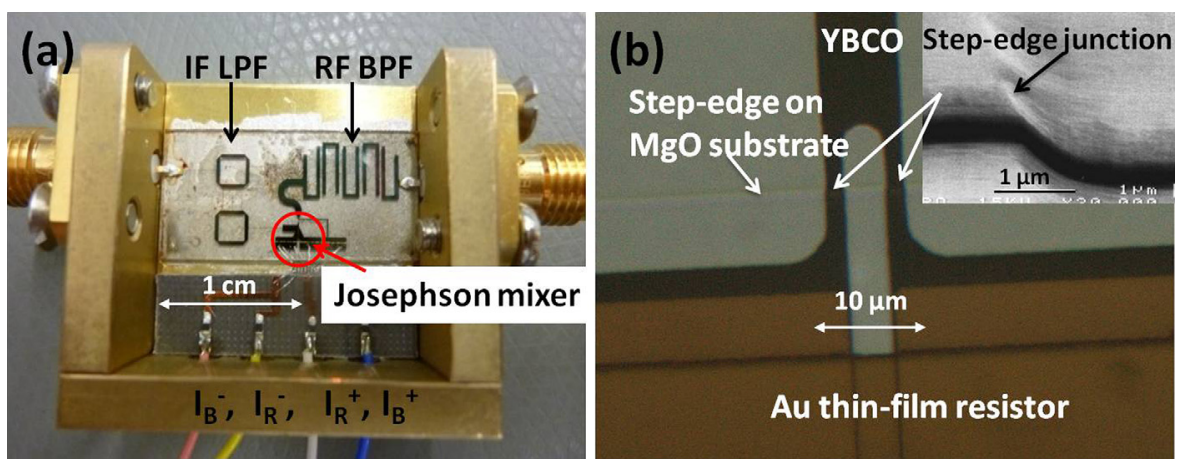

FIG. 1. (a) Photographs of the packaged monolithic HTS down-converter and (b) enlarged area of the heterodyne oscillator-mixer where two Josephson junctions are shunted by a $\mathrm{Au}$ thin-film resistor (the inset is a SEM micrograph of the YBCO step-edge junction on $\mathrm{MgO}$ substrate). oscillate at their intrinsic Josephson frequency, while the heterodyne frequency or the $\mathrm{LO}$ frequency $f_{\mathrm{LO}}$ appears at the difference frequency as determined by the differential voltage across $R_{\mathrm{s}}$. By adjusting the control current $I_{\mathrm{R}}$, the LO frequency can be tuned according to the Josephson frequency-voltage relationship

$$
f_{L O}=\frac{2 e}{h}\left(V_{0}+I_{R} R_{S}\right) .
$$

In the presence of an input RF signal, a down-converted intermediate frequency $f_{\mathrm{IF}}$ is produced and the single-sideband IF frequency $f_{\mathrm{IF}}$ can be written as

$$
f_{I F}=f_{R F}-\frac{2 e}{h}\left(V_{0}+I_{R} R_{S}\right)
$$

which is also tuneable by adjusting $I_{\mathrm{R}}$. The coefficient $2 e / h$ $\equiv 1 / \phi_{0}=0.4836 \mathrm{GHz} / \mu \mathrm{V}$ ( $\phi_{0}$ is the flux quantum) is the Josephson constant. $V_{0}$ is the off-set voltage at $I_{\mathrm{R}}=0$ due to the difference of the voltage drop across the two junctions at the biasing point $I_{\mathrm{B}}$.

The monolithic HTS down-converter module was fabricated on a $20 \mathrm{~mm} \times 10 \mathrm{~mm} \times 0.5 \mathrm{~mm} \mathrm{MgO}$ substrate using the CSIRO-developed step-edge junction technology. ${ }^{11,12}$ The creation of the step-pattern on the $\mathrm{MgO}$ substrate and patterning of the YBCO film were done using photolithographic and Ar ion beam etching techniques (more details can be found in Refs. 5, 6, and 10). For the device used in this experiment, the Au film thickness is $\sim 500 \mathrm{~nm}$ and the

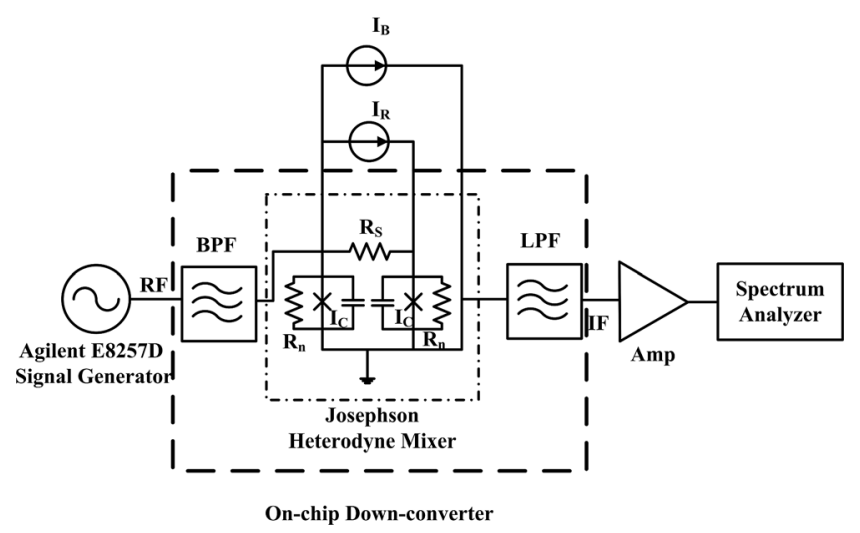

FIG. 2. Schematic diagram of the HTS MMIC frequency down-converter and measurement set-up where the larger dashed box includes the on-chip components and the smaller dashed box shows the Josephson heterodyne oscillator-mixer device. gap dimension is $4 \mu \mathrm{m} \times 120 \mu \mathrm{m}$ giving an estimated $R_{\mathrm{s}} \sim 4$ $\mathrm{m} \Omega$. The HTS circuit (depicted in Fig. 1) is more compact than any other reported hybrid or on-chip HTS downconverters of comparable frequency range and even more so compared to the conventional down converter. The fabricated down-converter was packaged in a copper thermal housing (see Fig. 1) and cooled down by a pulse-tube cryocooler. ${ }^{13}$ The microwave RF signal of frequencies between 7 and $9.5 \mathrm{GHz}$ generated by an Agilent Technologies E8257D signal generator was applied to the device via a cryogenic coaxial cable connected to the RF port of the MMIC chip. The IF outputs were taken out via the on-chip LPF, amplified by a room-temperature low-noise amplifier (LNA) of $26 \mathrm{~dB}$ gain, and displayed on an Agilent E4407B spectrum analyser. A transmission calibration of cable loss has been carried out up to the SMA ports by connecting a $50 \Omega$ stripline in place of the HTS chip. The conversion gain was obtained by subtracting the ratio $P_{\mathrm{IF}} / P_{\mathrm{RF}}$ from the cable loss and LNA gain.

Fig. 3 shows a linear relationship of the heterodyne oscillator frequency against the control current $I_{\mathrm{R}}$, described by Eq. (1) which enabled frequency tuneability from 0.1 to $7 \mathrm{GHz}$ and the exact value of $R_{\mathrm{S}}$ to be determined from the slope $R_{\mathrm{S}}=\left(\Delta f / \Delta I_{\mathrm{R}}\right) /(2 \mathrm{e} / h)=3.86 \mathrm{~m} \Omega$.

The IF output (or conversion efficiency) is bias $\left(I_{\mathrm{B}}\right)$ dependent as the non-linearity of the Josephson junction is a function of $I_{\mathrm{B}}$. Fig. 4 shows the conversion gain versus $I_{\mathrm{B}}$ at $30 \mathrm{~K}$ for $f_{\mathrm{LO}}=4.435 \mathrm{GHz}, f_{\mathrm{RF}}=8.4 \mathrm{GHz}$, and $f_{\mathrm{IF}}=3.965 \mathrm{GHz}$. The maximum conversion gain of $-14.7 \mathrm{~dB}$ is obtained at

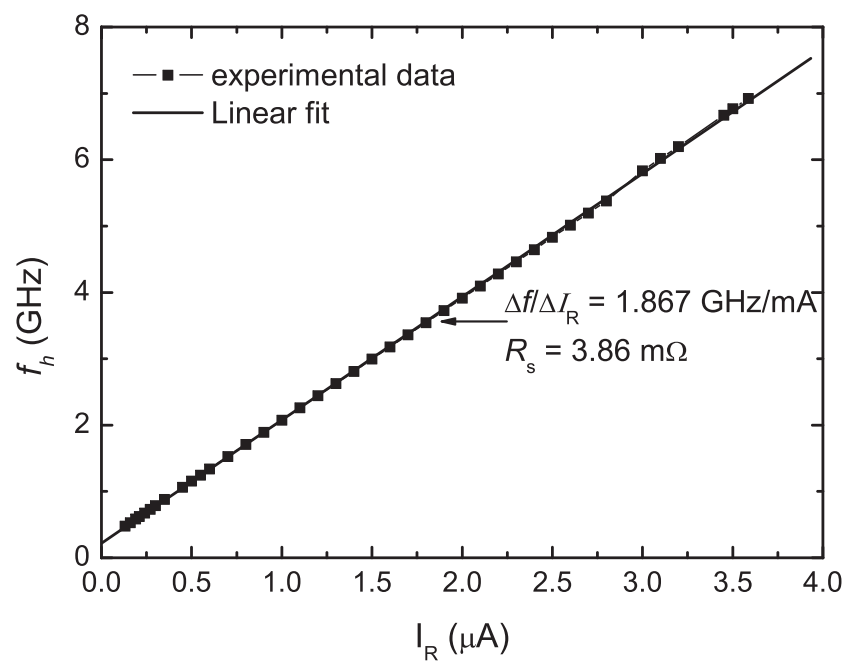

FIG. 3. Heterodyne oscillator frequency versus the control current $I_{\mathrm{R}}$; tuneable from 0.1 to $7 \mathrm{GHz}$ at $30 \mathrm{~K}$. 


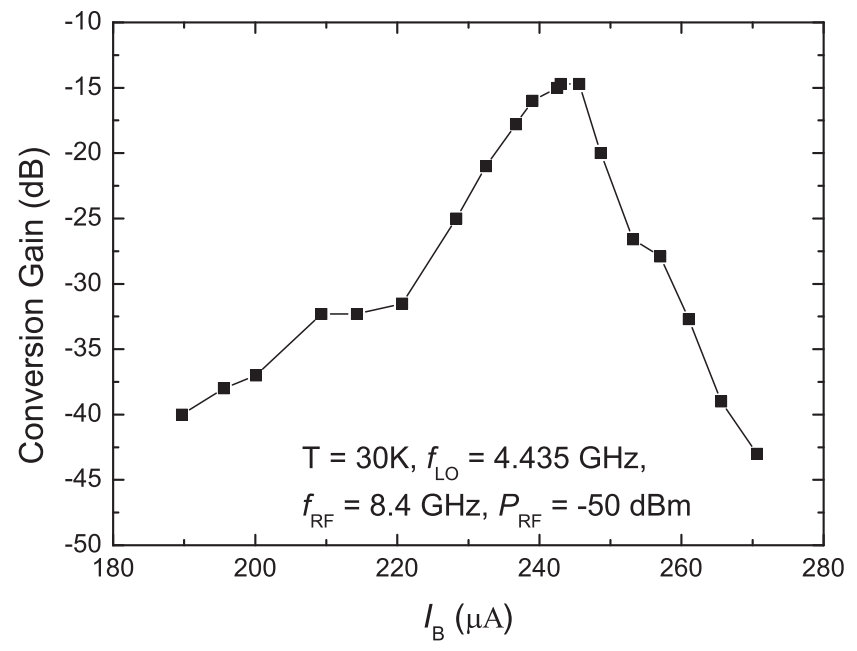

FIG. 4. Conversion gain as a function of bias current $I_{\mathrm{B}}$ at $30 \mathrm{~K}$ for a RF signal of $8.4 \mathrm{GHz}$ and $-50 \mathrm{dBm}$, and $f_{\mathrm{LO}}=4.435 \mathrm{GHz}$.

$I_{\mathrm{B}} \approx 240 \mu \mathrm{A}$ (just above the junction critical current $2 I_{\mathrm{c}}$ ). In some cases (e.g., for different RF frequencies, powers and different temperatures), multiple maxima of $P_{\mathrm{IF}}$ against $I_{\mathrm{B}}$ have been observed, ${ }^{10}$ due to the dependence of the single Josephson junction oscillations, the heterodyne LO oscillation, and, therefore the mixer IF output on $I_{\mathrm{B}}$. Further simulation work is underway to better understand the mixing mechanism.

Clear IF responses have been obtained at operating temperatures ranging from $15 \mathrm{~K}$ to $70 \mathrm{~K}$ with higher IF outputs occurring at lower temperatures. For clarity, only the $30 \mathrm{~K}$ results are presented in this paper. The frequency response of the HTS down-converter is shown in Fig. 5, where the RF input power is $-50 \mathrm{dBm}$. The heterodyne oscillator frequency was set to be $4.84 \mathrm{GHz}$ and the measured internal LO power is $-70 \mathrm{dBm}$. The frequency response corresponds to the RF BPF's transmission response; the IF output drops sharply outside of the RF pass band, although there is a slight frequency shift $(\sim 0.3 \mathrm{GHz})$ towards higher frequency, presumably due to slightly different dielectric constants of the $\mathrm{MgO}$ substrates used.

The operating range, linearity, and saturation of the down-converter were studied. Fig. 6 shows the experimental

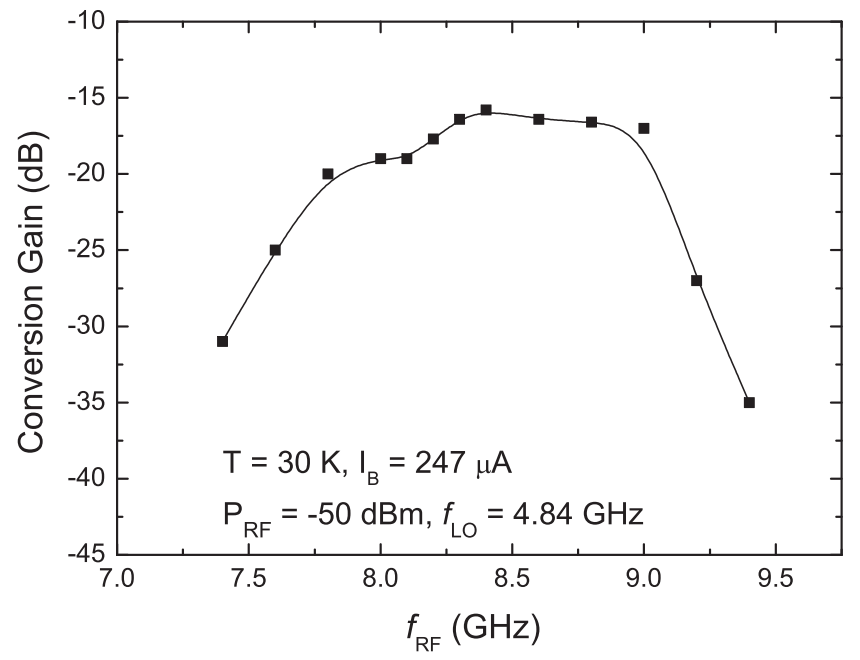

FIG. 5. Frequency response of the MMIC down-converter IF output (conversion gain).

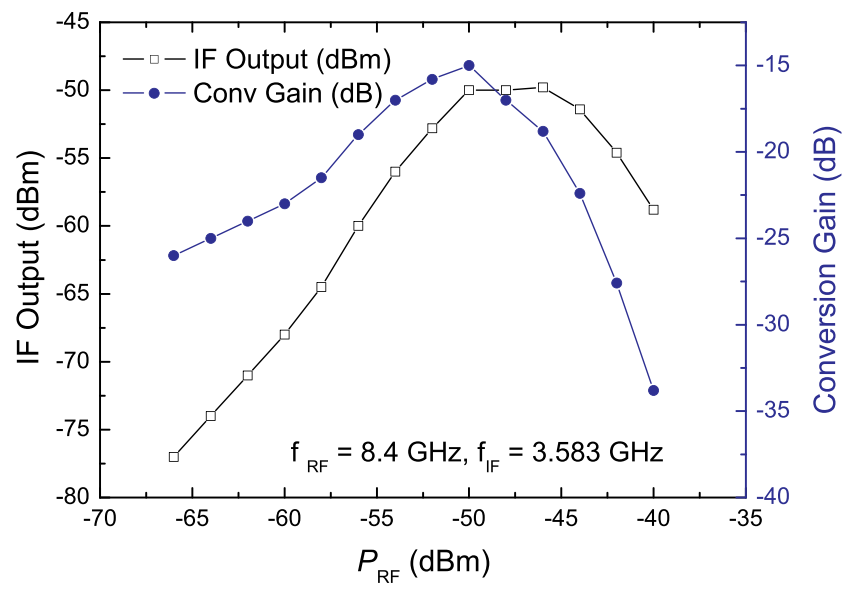

FIG. 6. IF output power and conversion gain as function of the RF signal power at $\mathrm{T}=30 \mathrm{~K}$.

result for a $\mathrm{RF}$ signal at $8.4 \mathrm{GHz}, f_{\mathrm{IF}}=3.583 \mathrm{GHz}$, and operating temperature of $30 \mathrm{~K}$. The IF output power increases linearly with $P_{\mathrm{RF}}$ from $-66 \mathrm{dBm}$ to $-50 \mathrm{dBm}$ and peaks at $P_{\mathrm{RF}} \sim-46 \mathrm{dBm}$. Further increase in the RF power suppresses the junction critical current, causing the internal LO amplitude, and therefore IF output, to fall. This behaviour was also shown by simulation and experiment on the earlier single Josephson device. ${ }^{10}$ By subtracting the ratio $P_{\mathrm{IF}} / P_{\mathrm{RF}}$ from the cable loss and LNA gain, the conversion gain is obtained (also plotted in the graph). A maximum conversion gain of $-15 \mathrm{~dB}$ is obtained from this graph. Note that the conversion loss includes the losses of the on-chip filters and coupling lines in addition to the Josephson mixer. This is a promising result given that no external LO is used and the measured internal Josephson heterodyne oscillation power is as low as $-70 \mathrm{dBm}$. The result is comparable to that of the HTS down-converter using an external LO source reported by Suzuki et al., ${ }^{14}$ where a maximum conversion gain of $-14.5 \mathrm{~dB}$ was obtained at $15 \mathrm{~K}$ with an LO power of $-18 \mathrm{dBm}$. There is scope for further improvement by optimizing the Josephson device and the impedance matching between the Josephson mixer and the input and output circuits.

The effective noise temperature $T_{m}$ of the device can be estimated from measuring the linewidth $\Delta f$ of the downconverter using the method described in Ref. 5. The intrinsic linewidth of the heterodyne oscillation is determined by the Johnson noise in $R_{\mathrm{s}}$

$$
\Delta f=\frac{4 \pi k T R_{s}}{\phi_{0}^{2}}
$$

which is temperature $T$ and $R_{\mathrm{s}}$ dependent. The effective noise temperature can be defined by rewriting Eq. (3) as

$$
T_{\text {noise }}=\frac{\Delta f \phi_{0}^{2}}{4 \pi k R_{s}},
$$

where $\Delta f$ and $R_{\mathrm{s}}(3.86 \mathrm{~m} \Omega$ for this device) are experimental values. We measured the linewidths $\Delta f$ for both the LO and IF peaks at various temperatures and converted to the $T_{\text {noise }}$ using Eq. (4). The results are plotted in Fig. 7. Within experimental scatter, it is clear that the effective noise temperature of the described monolithic down-converter is proportional 


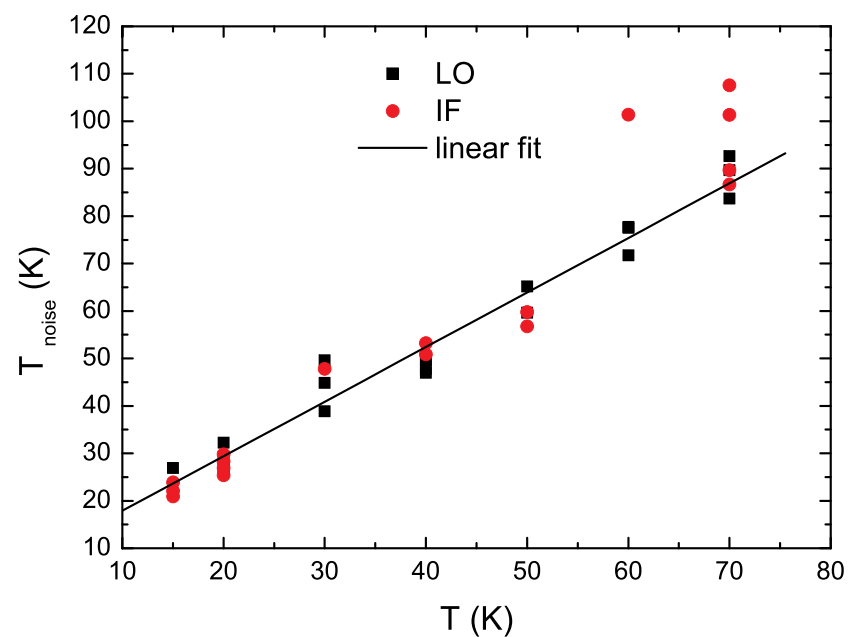

FIG. 7. Effective noise temperature versus physical temperature.

to, but $10-20 \mathrm{~K}$ greater than, the physical temperature over the measured range. Greater scatter at $\mathrm{T} \geq 60 \mathrm{~K}$ may indicate additional noise contribution other than the thermal noise of the shunt resistor $R_{\mathrm{S}}$. The total receiver noise temperature or noise, however, is expected to be higher and will be more precisely measured in ongoing experiments.

In summary, a compact monolithic HTS down-converter has been demonstrated. The on-chip HTS circuit consists of a 7-9 GHz RF BPF, a 0-4 GHz IF LPF, and a self-pumped frequency-tuneable Josephson heterodyne mixer on a $20 \mathrm{~mm} \times 10 \mathrm{~mm} \mathrm{MgO}$ substrate. The frequency response corresponds well with that of the designed RF signal BPF filter. A conversion gain (including all on-chip components) of around $-15 \mathrm{~dB}$ is obtained, which is very encouraging as there was no external LO used but only a low internal oscil- lator power of $-70 \mathrm{dBm}$. The results of bias dependence, dynamic range, and linearity as well as the effective noise temperature were presented. The described on-chip HTS down-converter has the features of compactness, low loss, and low power consumption, which are very attractive for applications in receiver front-ends of wireless communication systems.

We acknowledge technical assistance from Mei Shen, Jeina Lazar, and Chris Williams of CSIRO and helpful discussion and advice from Dr. Colin Pegrum of University of Strathclyde and Dr. Yong Cai of CSIRO.

${ }^{1}$ R. R. Mansour, IEEE Trans. Microwave Theory Tech. 50, 750 (2002).

${ }^{2}$ R. W. Simon, R. B. Hammond, S. J. Berkowitz, and B. A. Willemsen, Proc. IEEE 92, 1585 (2004).

${ }^{3}$ X. Zhang, Q. Meng, F. Li, C. Li, S. Li, A. He, H. Li, and Y. He, Supercond. Sci. Technol. 19, S394 (2006).

${ }^{4}$ N. Klein, Rep. Prog. Phys. 65, 1387 (2002).

${ }^{5}$ J. Du, J. C. Macfarlane, and L. Hao, Appl. Phys. Lett. 93, 033507 (2008).

${ }^{6}$ J. C. Macfarlane, J. Du, R. Taylor, and C. M. Pegrum, IEEE Trans. Appl. Supercond. 19, 920 (2009).

${ }^{7}$ C. M. Pegrum, J. C. Macfarlane, and J. Du, IEEE Trans. Appl. Supercond. 21, 349 (2011).

${ }^{8}$ J. Du and J. C. Macfarlane, Electron. Lett. 47, 772 (2011)

${ }^{9}$ J. Du, J. C. Macfarlane, T. Zhang, Y. Cai, and Y. J. Guo, Supercond. Sci. Technol. 25, 025019 (2012).

${ }^{10}$ J. Du, J. C. Macfarlane, C. M. Pegrum, T. Zhang, Y. Cai, and Y. J. Guo, J. Appl. Phys. 111, 053910 (2012).

${ }^{11}$ C. P. Foley, E. E. Mitchell, S. K. H. Lam, B. Sankrithyan, Y. M. Wilson, D. L. Tilbrook, and S. J. Morris, IEEE Trans. Appl. Supercond. 9, 4281 (1999).

${ }^{12}$ E. E. Mitchell and C. P. Foley, Supercond. Sci. Technol. 23, 065007 (2010).

${ }^{13}$ J. Du, J. C. Macfarlane, S. H. K. Lam, and R. Taylor, Supercond. Sci. Technol. 22, 105013 (2009).

${ }^{14}$ K Suzuki, S. Yoshikawa, K. Yamaguchi, K. Hayashi, S. Fujino, T. Takenaka, T. Mitsuzuka, Y. Enomoto, K. Imai, F. Suginoshita, and N. Yazawa, IEEE MTT-S Int. Microwave Symp. Dig. 1993, 1429. 